[33] S. C. Yang, A. Freedman, M. Kawasaki, and R. Bersohn, "Energy distribution of the fragments produced by photodissociation of $\mathrm{CS}_{2}$ at $193 \mathrm{~nm}, " J$. Chem. Phys., vol. 72, p. 4058, 1980.

[34] A. Gedanken and M. D. Rowe, "Negative circular docjroism of the methyl halides. Resolution of the $n \rightarrow \sigma^{*}$ continuum," Chem. Phys. Lett., vol. 34, p. 39, 1975.

[35] H. Okabe, Photochemistry of Small Molecules. New York: Wiley, 1978.

[36] M. Kawasaki, S. J. Lee, and R. Bersohn, "Photodissociation of molecular beams of methyl iodide and iodoform," $\mathrm{J}$. Chem. Phys., vol. 63, p. 809, 1975.

[37] P. M. Kroger, P. C. Demou, and S. J. Riley, "Polyhalide photofragment spectra. I. Two-photon two-step photodissociation of methylene iodide,"J. Chem. Phys., vol. 65, p. 1823, 1976.

[38] R. K. Lengel and R. N. Zare, "Experimental determination of the singlet-triplet splitting in methylene," J. Amer. Chem. Soc., vol. 100 , p. $7495,1978$.

[39] D. Feldmann, K. Meier, H. Zacharias, and K. H. Welge, "The threshold for photodissociation of ketene into $\mathrm{CH}_{2}\left(\widetilde{\mathrm{a}}^{\mathrm{P}} \mathrm{A}_{1}\right)$ and the $\mathrm{CH}_{2}\left(\tilde{\mathrm{a}}^{1} \mathrm{~A}_{1}-\tilde{\mathrm{X}}^{3} \mathrm{~B}_{1}\right)$ energy separation," Chem. Phys. Lett., vol. 59 , p. 171,1978 .

[40] A. Freedman and R. Bersohn, "Photodissociation of molecular beams. Cleavage of metal-metal bonds in rhenium and manganese decacarbonyl," J. Amer. Chem. Soc., vol. 100, p. 4116, 1978.

[41] M. Dzvonik, S. Yang, and R. Bersohn, "Photodissociation of mo- lecular beams of aryl halides," J. Chem. Phys., vol. 61, p. 4408, 1974.

[42] A. Freedman, S. C. Yang, M. Kawasaki, and R. Bersohn, "Energy distribution of the fragments produced by photodissociation of $\mathrm{CS}_{2}$ at $193 \mathrm{~nm}, " J$. Chem. Phys., vol. 72, p. 4058, 1980.

[43] R. Bersohn, "Photodissociation spectroscopy: Symmetry and lifetime of the excited state," Istrum. J. Chem., vol. 14, p. 111, 1975.

[44] K. Shobatake, R. K. Sparks, L. R. Carlson, and Y. T. Lee, presented at the XI ICPEAC Conf., Kyoto, Japan, 1979.

[45] W. G. Hawkins and P. L. Houston, "193 nm photodissociation of $\mathrm{H}_{2}$ S: The SH internal distribution," J. Chem. Phys., vol. 72, p. $297,1980$.

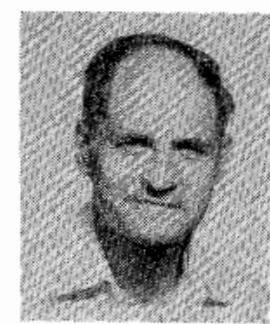

Richard Bersohn was born in New York, NY, in 1925. He received the B.S. degree from the Massachusetts Institute of Technology, Cambridge, MA, in 1943, and the Ph.D. degree from Harvard University, Cambridge, MA, in 1950.

From 1951 to 1959 he taught at Cornell University, Ithaca, NY. Presently he is with Columbia University, New York, NY.

Dr. Bersohn is a member of the American Physical Society.

\title{
Application of Laser Photolysis to the Study of Reactive Intermediates
}

\author{
NICHOLAS J. TURRO, MASAYUKI AIKAWA, AND JARED A. BUTCHER, JR.
}

\begin{abstract}
Excimer laser flash photolysis in the study of diphenyl carbene (DPC) reveals the presence of at least three transient species produced by excitation of a precursor at $249 \mathrm{~nm}$ in isooctane solution at room temperature depending on the concentration of $\mathrm{O}_{2}$ present. In an aerated solution, a fast first-order decay assigned to ${ }^{3} \mathrm{DPC}$ leads to the formation of an unassigned species which also decays by first order kinetics to produce the observed product $\mathrm{Ph}_{2} \mathrm{CO}$. In degassed solution, the fast first-order decay of a species to produce ${ }^{3} \mathrm{DPC}$ which decays by second-order kinetics to produce the dimeric products observed.
\end{abstract}

Manuscript received February 19, 1980; revised June 23, 1980.

The authors are with the Department of Chemistry, Columbia University, New York, NY 10027.

\section{INTRODUCTION}

$T$ THE development of excimer lasers at last permits photochemists to study chemical systems using high power excitation at short wavelengths, greatly enlarging the number of problems amenable to study by this technique. The advantages of using lasers are twofold: 1) the high photon flux can produce a high concentration of excited species in a very short time; 2) multiphotonic processes can lead to photochemistry of these very short lived species, presenting the possibility of observing new products which are peculiar to laser induced photochemistry. In addition, when coupled with state of the art detection, laser induced fluorometry can be 
TRANSIENT ABSORPTION AND EMISSION DETECTION SYSTEM

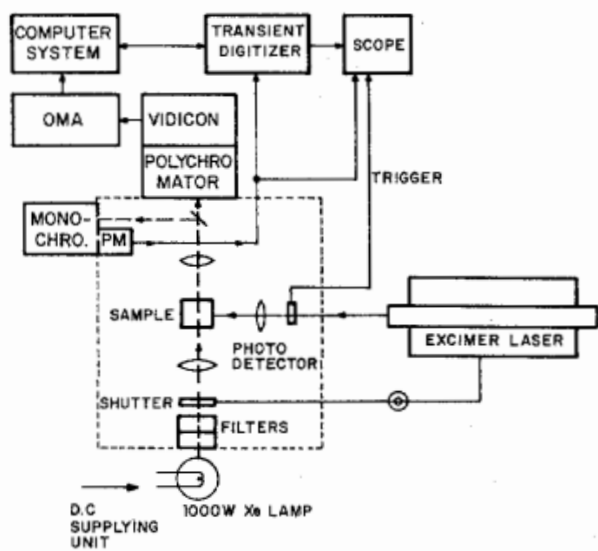

Fig. 1. Transient absorption and emission detection system.

beneficial in elucidating mechanisms by the detection of the emission of transient species as well as stable products at extremely low concentration. Recent work in our laboratory reveals the advantages of laser photolysis and points the way to a future of fruitful research utilizing lasers to initiate novel photochemical processes in complex molecules. (Since production of specialty chemicals such as drugs is at present the most economically feasible application of excimer lasers, ground-breaking work in this area has practical implications in addition to being of scientific import.)

\section{EXPERIMENTAL SETUP}

The flash system currently being employed consists of a Lambda Physik EMG 500 excimer laser, a standard nanosecond flash setup with the additional capability of direct spectral analysis by OMA (see Fig. 1). The optical system is enclosed in a black box to permit emission studies as well as absorption. The cell holder is of significant importance. In addition to the traditional requirements of optical alignment, temperature control, and so forth, a cell holder for laser studies should be flexible in regard to the longitudinal displacement of the cell to permit observation not only of the body of the cell but also the region at the very front ( 1 or 2 $\mathrm{mm}$ ). The reason for this comes out of the requirement that in the case of an absorption experiment it is necessary to have a sufficient concentration of the absorbing transient to separate the absorption from the noise, while simultaneously maintaining a low enough concentration of the starting material that the entire region being probed is photolyzed.

\section{Appropriate Chemical Systems}

Two systems-diphenyl carbene (DPC) and diphenyl trimethylene methane (DPTMM)-will serve to exemplify the potential of these techniques and also as a comparison of existing technologies in this area. Using the setup described above, we have obtained data regarding the spectral and temporal properties of these transient species. Both DPC and DPTMM are ground-state triplet species, and they are well studied at $77 \mathrm{~K} \mathrm{[1].} \mathrm{Both} \mathrm{species} \mathrm{exhibit} \mathrm{strong} \mathrm{absorptions} \mathrm{in} \mathrm{the}$ 320-330 $\mathrm{nm}$ region without an accompanying emission band, as well as a weak absorption at $450-480 \mathrm{~nm}$ which exhibits mirror symmetry with an emission $\sim 500 \mathrm{~nm}$ at $77 \mathrm{~K}$. In the absence of a quencher, both lead to dimeric products. Thus, we anticipated similar behavior for the two systems.

\section{EXPERIMENTAL}

\section{Materials}

Diphenyldiazomethane was prepared in-house and found to be free of $\mathrm{Ph}_{2} \mathrm{CO}$ by IR. Tetraphenyl oxirane was prepared by literature procedures and crystallized twice from ethanol before use. Other precursors were generously provided by Griffin and Berson and used without additional purification. Isooctane, Gold Label Aldrich, was used without further purification. Comparison to authentic samples: $\underline{6}, \underline{7}$, and $\underline{8}$ (Aldrich) and $\underline{5}$ by photolysis of $\underline{8}$ [2], purified as required.

\section{Emission Data: Diphenyl Carbene (DPC)}

As shown in (1), diphenyl carbene can be formed from both

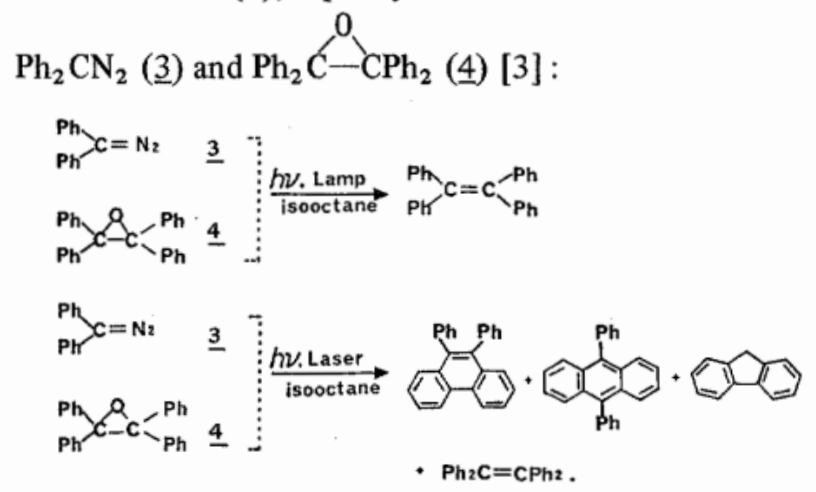

The principal product under normal lamp photolysis is tetraphenylethylene, $\mathrm{Ph}_{2} \mathrm{C}=\mathrm{CPh}_{2}$, or benzophenone azine, $\mathrm{Ph}_{2} \mathrm{C}=\mathrm{N}-\mathrm{N}=\mathrm{CPh}_{2}$, depending on the starting material [4] . Using a laser, however, the formation of $\mathrm{Ph}_{2} \mathrm{C}=\mathrm{CPh}_{2}$ is remarkably enhanced and the formation of the azine suppressed. In addition, as shown in (2), new compounds never before reported as photochemical products are formed in trace amounts (1 percent) at room temperature at high laser power (100 mJ/pulse at $249 \mathrm{~nm}, 15 \mathrm{~ns}$ FWHM, in a $0.3 \mathrm{~cm}^{2}$ area). The product distribution also depends heavily on the concentration of the carbene, laser power, and the presence of $\mathrm{O}_{2}$.

Diphenyl carbene exemplifies a case in which circumstances combine to form a complete package easily interpreted by first order analysis. The products of interest emit strongly at room temperature and can be recorded in the presence of high concentrations of $\mathrm{Ph}_{2} \mathrm{C}=\mathrm{CPh}_{2}$ which emits only weakly. Thus, the presence of $\underline{5}, \underline{6}$, and $\underline{7}$ could be easily detected and identified spectroscopically. This is shown in Figs. 2-4. Further verification of the products was obtained using TLC and GC analysis and comparison to authentic samples.

\section{Absorption Data}

Absorption spectra of short-lived species can be obtained using OMA technology in which the vidicon serves in the capacity of a photographic plate; however, usable time resolution is difficult to obtain. Fig. 5(a) shows an OMA absorption spectrum of DPC. Consequently, the most reliable method for obtaining time resolved absorption spectra in the nanosecond 


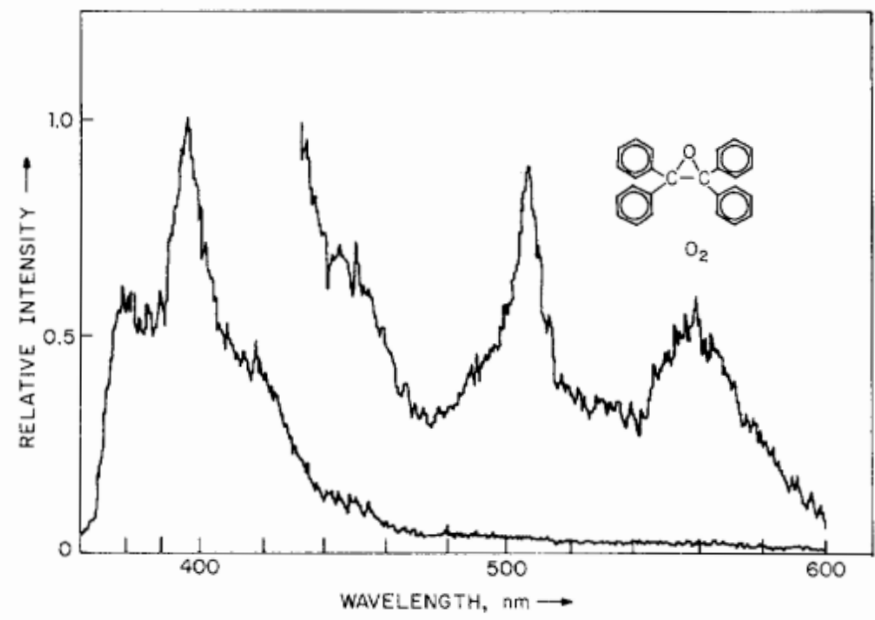

Fig. 2. Emissive products formed during laser photolysis in aerated solution as observed by OMA.

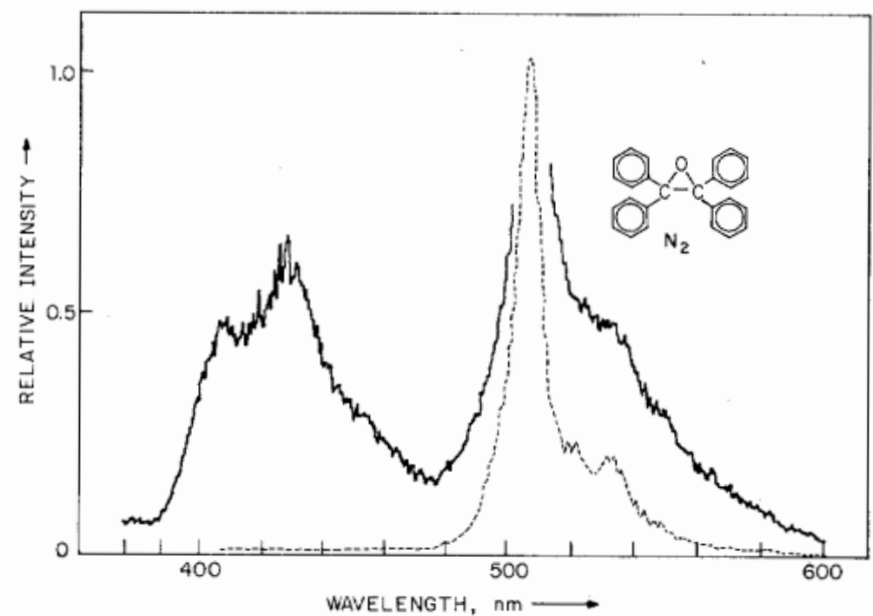

Fig. 3. Emissive products formed during laser photolysis in $\mathrm{N}_{2}$ purged solution as observed by OMA.

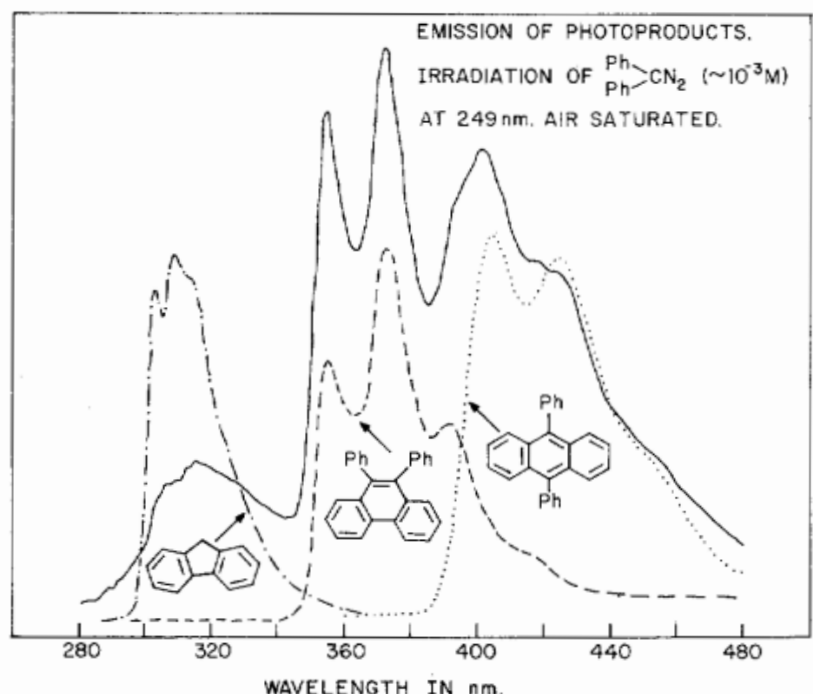

Fig. 4. Emissive products identified by comparison to authentic samples.

time regime is to obtain absorption decays at a series of wavelengths and plot the time resolved spectra "point by point." A serious problem is the pulse to pulse variation in laser power. Fig. 5(b) shows such a series of spectra taken at 5

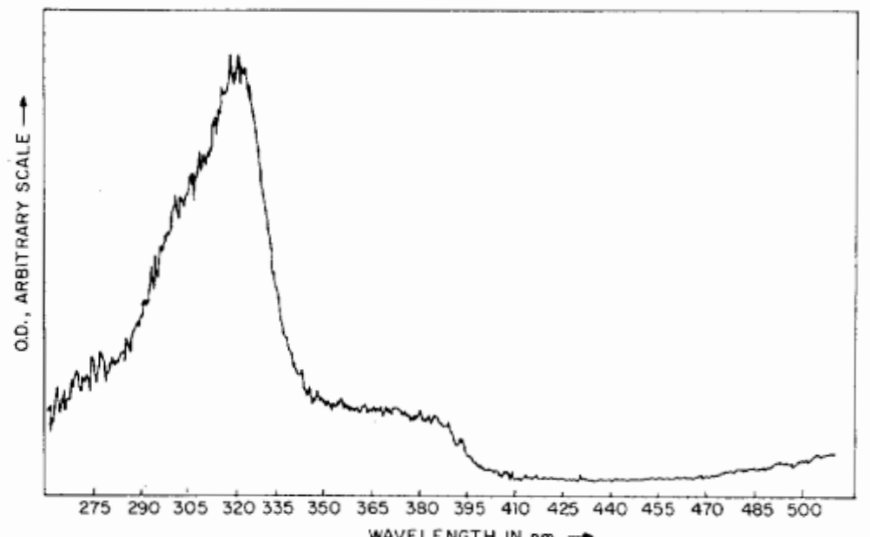
WAVELENGTH IN $\mathrm{RM}$

(a)

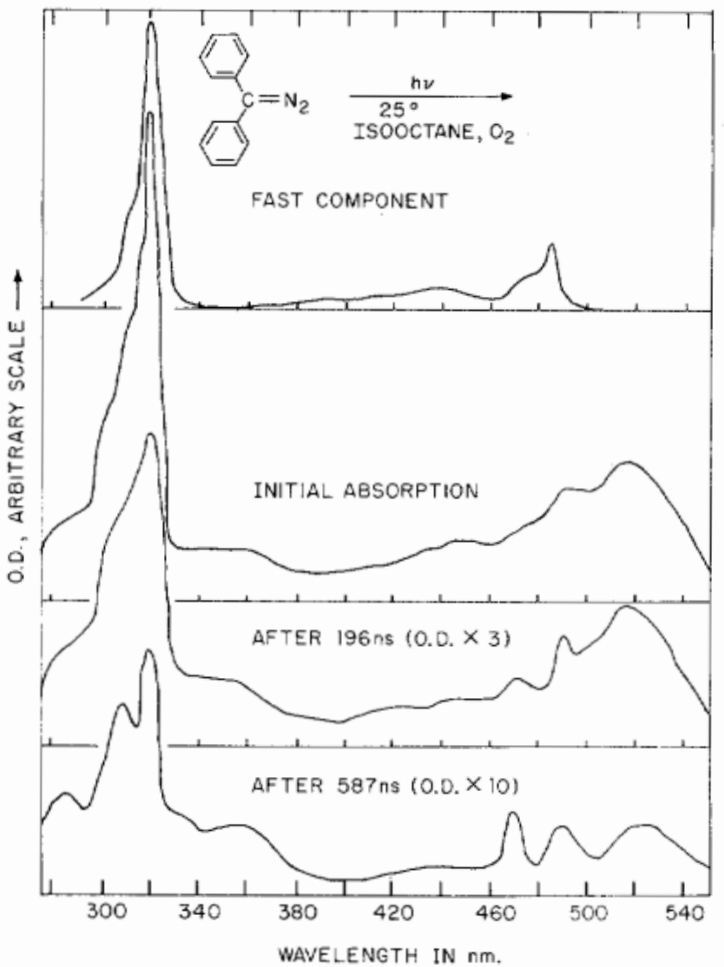

(b)

Fig. 5. (a) Transient absorption spectrum of diphenyl carbene in aerated isooctane recorded at room temperature by optical multichannel analyzer (OMA). (b) Time resolved absorption spectra for DPC in aerated isooctane at room temperature.

$\mathrm{nm}$ resolution for DPC neglecting laser power fluctuations. Significantly, the spectrum obtained immediately following the laser pulse in the $500 \mathrm{~nm}$ range differs from that assigned to DPC. Careful examination of the decay in aerated solution revealed the presence of a very short-lived component in the decay, and the point by point plot of this very rapidly decaying component yields the familiar absorption spectrum of DPC as witnessed by the top trace in Fig. 5. In light of this discovery we are at present unwilling to assign a structure to the longer lived component. That it might be a radical species or carbonyl oxide has not escaped our notice, and experiments designed to test these possibilities are in progress.

Upon degassing, a remarkable change in the absorption spectrum occurs. Not only does the intensity of the absorption drop significantly at all wavelengths, spectral changes occur. The maximum absorption assigned to ${ }^{3} \mathrm{DPC}$ occurs at 320 and $480 \mathrm{~nm}$ and this is observed in the aerated solution. 


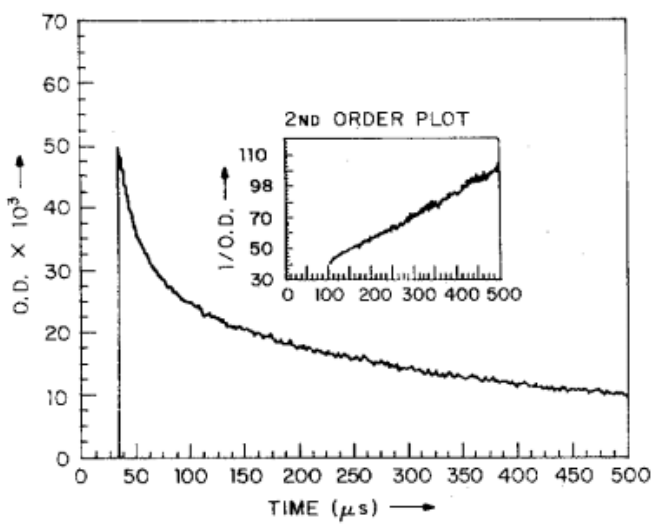

Fig. 6. Second-order decay of transient observed at $320 \mathrm{~nm}$ in degassed solution.

The maximum absorptions in degassed solution are at 340 and $525 \mathrm{~nm}$ : thus we must conclude that the species observed is something other than ${ }^{3} \mathrm{DPC}$. Some data on the nature of this transient have been obtained from the absorption kinetics.

\section{Absorption Kinetics}

Analysis of the decay of the absorption due to a transient species observed at a given wavelength was straightforward. The raw data were converted to $O D$ using the algorithm

$$
O D=\log \left(\frac{I(O)}{I(t)}\right)
$$

where $I(O)$ is the intensity prior to the laser flash and $I(t)$ is recorded by the transient digitizer during the decay. The data were tested for first- and second-order behavior by plotting $\log O D$ and $1 / O D$, respectively.

At $320 \mathrm{~nm}$ in the aerated solution, two first-order decays are observed ( $\tau_{\text {fast }}=15 \mathrm{~ns}, \tau_{\text {slow }}=200 \mathrm{~ns}$ ). This is the $\lambda_{\max }$ for the ${ }^{3}$ DPC and the fast component is assigned to this species. The species absorbing less than $500 \mathrm{~nm}$ exhibits a build-up in absorption in which the lifetime corresponds to the rapid decay revealed at $320 \mathrm{~nm}$. We take this as evidence that this species is an unstable product derived from ${ }^{3} \mathrm{DPC}$ which itself undergoes a first-order decay, e.g., the carbonyl oxide, 9 .

In the degassed solution an extremely unusual situation is observed. At short times, when the maximum concentration of transient species is anticipated, second-order decay is recorded at 320 and $480 \mathrm{~nm}$, maxima in the ${ }^{3} \mathrm{DPC}$ absorption (see Fig. 6); but at 360 and $525 \mathrm{~nm}$, where the ${ }^{3} \mathrm{DPC}$ does not absorb significantly, first-order kinetics are observed. At intermediate wavelengths, e.g., $350 \mathrm{~nm}$, fast first-order $(\tau=$ $5.8 \mu \mathrm{s}$ ) and slow second-order decays are observed (see Fig. 7). The latter second-order decay is identical to that previously observed by Closs and Rabinow [4] and, furthermore, if extrapolated to zero time this second-order component exhibits maxima at 320 and $480 \mathrm{~nm}$ and is thus assigned to ${ }^{3}$ DPC. The identity of the rapidly decaying species observed under degassed conditions remains a mystery.

Fig. 8 shows a speculative working hypothesis which explains the production of the unusual products we observed and is consistent with all of the data obtained to date. In the presence of $\mathrm{O}_{2},{ }^{1} \mathrm{DPC}$ undergoes irreversible intersystem crossing to ${ }^{3} \mathrm{DPC}$ which is trapped by $\mathrm{O}_{2}$ to form the carbonyl oxide $\underline{9}$ or some intermediate leading to the formation of (a)

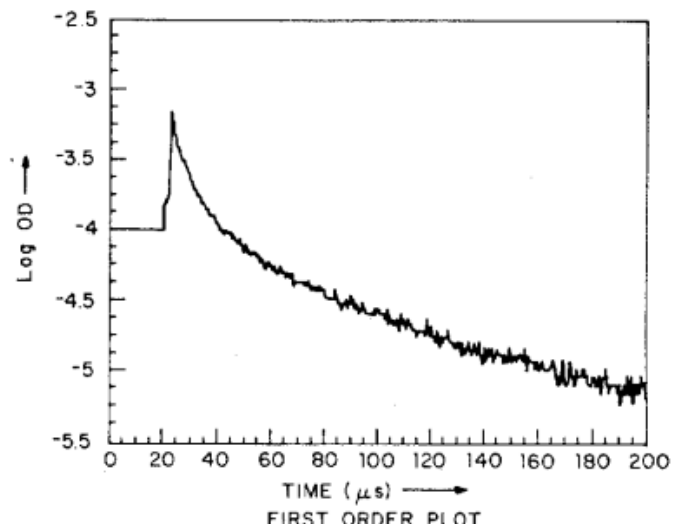

(b)

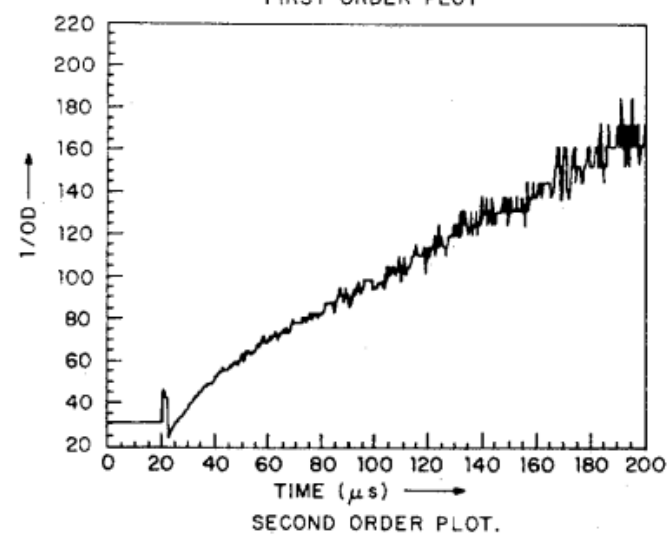

Fig. 7. Decay curves for DPC revealing the presence of (a) fast firstorder component and (b) slow second-order decay.

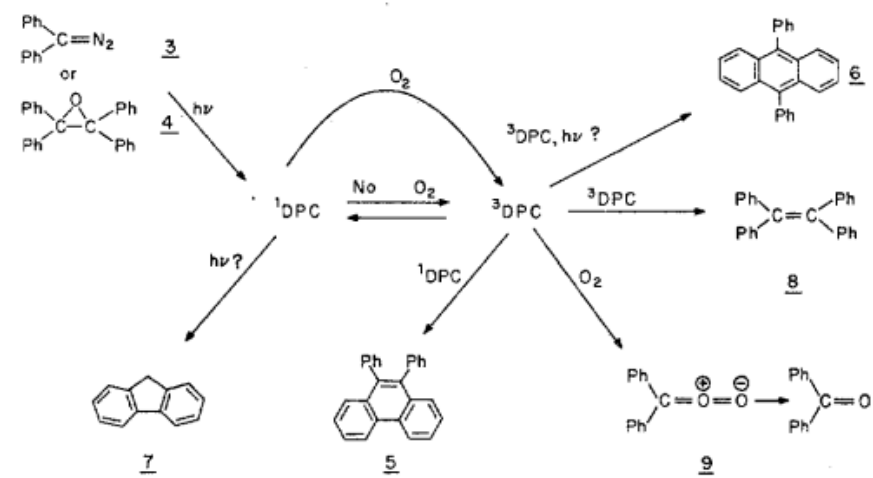

Fig. 8. Scheme I.

benzophenone which is the principle product under these conditions. In the absence of $\mathrm{O}_{2}$, a rapid equilibrium is set up between ${ }^{1} \mathrm{DPC}$ and ${ }^{3} \mathrm{DPC}$. We postulate that fluorine (7) arises from the photochemically excited ${ }^{1} \mathrm{DPC}$ since quenching of the production of fluorine and quenching of the intensity of the emission from the carbene by methanol are comparable. The source of the excited carbene, i.e., biphotonic excitation in which consecutive photons decompose the starting material and then excite the carbene, or monophotonic production of the excited carbene via an adiabatic photochemical route, remains as an uninvestigated problem. As to compounds $\underline{5}$ and $\underline{6}$, the number of photons required for their production is not a simple matter because of complications arising from internal filter effects which result from light absorption by products. Suffice it to say that Fig. 8 is consistent with our observation that they arise from different routes and that work in this area is continuing. 


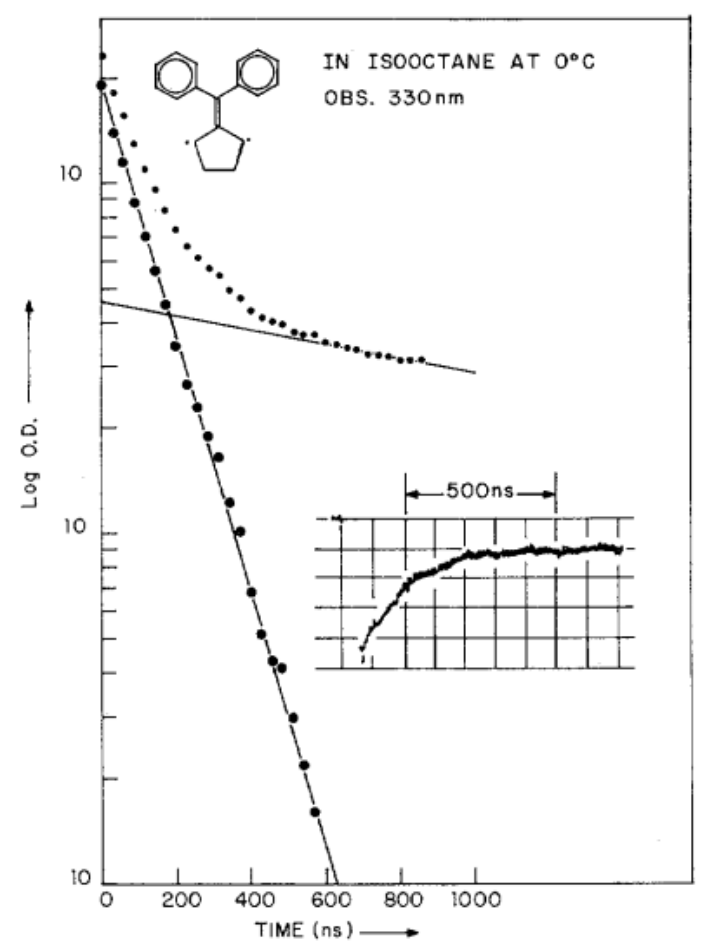

Fig. 9. Typical decay for $\phi_{2}$ TMM.

\section{Diphenyl Trimethylene Methane (DPTMM)}

As in the case of DPC, DPTMM has been well studied at 77 $\mathrm{K}$ with respect to its emission and absorption properties [1] : furthermore, laser excitation $(249 \mathrm{~nm})$ of the azo precursor at room temperature in isooctane produces the characteristic emission as recorded by OMA. Low resolution point-by-point absorption spectra, obtained as described above, were sufficiently consistent with the $77 \mathrm{~K}$ spectrum to justify a kinetics study at the absorption maximum $(330 \mathrm{~nm})$. Interestingly, the kinetics observed in the aerated solution were similar to those for degassed solutions of DPC in that they consisted of a fast first-order component ( $\tau=150 \pm 25 \mathrm{~ns})$ and a slow second-order decay (see Fig. 9). This result opens, as a viable speculation, the idea that intersystem crossing in this system may be measurably slow.

\section{ConClusions}

As shown previously, the combination of excimer laser excitation and OMA technology permits the detection of emissive products on the trace level and allows for the study of products heretofore unobserved at room temperature. Principal among these is DPC itself. The judicious combination of OMA detection and decay kinetics (absorption or emission) recorded with a transient digitizer allows for the detection and, in principle at least, the identification of species with a fleeting existence. The composite intensity, wavelength, and time profile gives information which would otherwise be missed. Through this means we have found that DPC is capable of diverse and interesting chemistry. It is our contention that a wealth of information about a number of systems can be had using excimer laser induced flash photolysis and state of the art detection.

\section{REFERENCES}

[1] A. M. Trozzolo and W. A. Gibbons, "The absorption, emission and excitation spectra of diarylmethylenes," J. Amer. Chem. Soc., vol. 89, pp. 239-243, Jan. 1967; N. J. Turro, M. J. Mirbach, N. Harrit, J. A. Berson, and M. S. Platz, "Evidence from the absorption and emission spectra of trimethylenemethane derivatives for two molecular species in thermal equilibrium," J. Amer. Chem. Soc., vol. 100, pp. 7653-7658, Nov. 1978.

[2] F. B. Mallory, C. S. Wood, and J. T. Gordon, "Photochemistry of stilbenes. III. Some aspects of the mechanism of photocyclization to phenanthrenes," J. Amer. Chem. Soc., vol. 86, pp. 30943102, Aug. 1964.

[3] R. S. Becker, R. O. Bost, J. Kolc, N. R. Bertoniere, R. L. Smith, and G. W. Griffin, "Spectroscopy and photochemistry of aryloxiranes," J. Amer. Chem. Soc., vol. 92, pp. 1302-1311, Mar. 1970.

[4] G. L. Closs and B. E. Rabinow, "Kinetic studies on diarylcarbenes," J. Amer. Chem. Soc., vol. 98, pp. 8190-8198, Dec. 1976.

Nicholas J. Turro, photograph and biography not available at the time of publication.

Masayuki Aikawa, photograph and biography not available at the time of publication.

Jared A. Butcher, Jr., photograph and biography not available at the time of publication. 